Draft Version March 14, 2018

Preprint typeset using $\mathrm{LAT}_{\mathrm{E}} \mathrm{X}$ style emulateapj v. 08/22/09

\title{
ECLIPSING BINARIES IN THE MACHO DATABASE: NEW PERIODS AND CLASSIFICATIONS FOR 3031 SYSTEMS IN THE LARGE MAGELLANIC CLOUD
}

\author{
A. Derekas ${ }^{1}$, L. L. Kiss and T. R. Bedding \\ School of Physics, University of Sydney, NSW 2006, Australia \\ Draft version March 14, 2018
}

\begin{abstract}
Eclipsing binaries offer a unique opportunity to determine fundamental physical parameters of stars using the constraints on the geometry of the systems. Here we present a reanalysis of publicly available two-color observations of about 6800 stars in the Large Magellanic Cloud, obtained by the MACHO project between 1992 and 2000 and classified as eclipsing variable stars. Of these, less than half are genuine eclipsing binaries. We determined new periods and classified the stars, 3031 in total, using the Fourier parameters of the phased light curves. The period distribution is clearly bimodal, reflecting refer to the separate groups of more massive blue main sequence objects and low mass red giants. The latter resemble contact binaries and obey a period-luminosity relation. Using evolutionary models, we identified foreground stars. The presented database has been cleaned of artifacts and misclassified variables, thus allowing searches for apsidal motion, tertiary components, pulsating stars in binary systems and secular variations with time-scales of several years.

Subject headings: binaries: eclipsing — galaxies: individual (Large Magellanic Cloud) — stars: statistics - stars: variables: other
\end{abstract}

\section{INTRODUCTION}

The last decade witnessed the birth of a new research field, the large-scale study of variable stars in external galaxies. This has been made possible by the huge databases of microlensing observations in the Magellanic Clouds, such as the MACHO (Alcock et al. 1993), OGLE (Udalski et al. 1997) and EROS (Aubourg et al. 1995) projects. These programs (beyond their primary purpose) resulted in the discovery of thousands of eclipsing binaries and many other types of variable stars and gave an unprecedented homogenous coverage of their light curves. Recent all-sky surveys also give a good opportunity to study large numbers of stars (Paczvński et al. 2006). However, processing the huge amount of data can be quite challenging, even when looking at seemingly simple issues such as classification of variables, period determination, etc.

The astrophysical potential of large databases of eclipsing binaries has been explored by a number of authors, but we are still far from a full exploitation. Catalogs of eclipsing variables in the Magellanic Clouds have been published with an increasing completeness (Alcock et al. 1997; Wvrzykowski et al. 2003, 2004). Attempts have been made to improve our understanding of the formation of contact binaries, especially those with giant components (Rucinski 1997a, b, 1998; Maceroni \& Rucinski 1999; Rucinski \& Maceroni 2001). To help analyse large samples of stars, various automated pipelines have been developed (Devor 2005; Tamuz et al. 2006; Mazeh et al. 2006), while the large-number statistics helped investigate orbital circularization over a broad range of stellar parameters (Faccioli et al. 2005). Another application is determining accurate distances using early-type detached eclipsing binaries, which has been shown to offer the most accurate calibration of the local distance scale (e.g., Guinan et al. 1998; Ribas et al. 2000; Wvithe \& Wilson

\footnotetext{
${ }^{1}$ E-mail: derekas@physics.usyd.edu.au
}

2001; Salaris \& Groenewegen 2002; Fitzpatrick et al. 2002; Ribas et al. 2002; Michalska \& Pigulski 2005).

In contrast to these applications, the near-decade long time coverage of the available data has rarely been utilised. For example, Palen \& Armstrong (2003) and Johnson et al. (2004) reported on searches for tertiary companions of eclipsing binaries in the MACHO database, but no definite results have been published.

Here we present an analysis of the publicly available MACHO light curves of LMC variables classified as eclipsing binaries, which have been online since 2001 . The main aim of this project is to measure period changes and search for eclipsing binaries with pulsating components. The period-luminosity $(\mathrm{P}-\mathrm{L})$ relations of contact binaries and their relation to pulsating red giants were already discussed in Derekas et al. (2006). In this paper we discuss the general properties of the sample, presenting a full reclassification, newly determined periods, the colormagnitude diagram and period-luminosity distributions.

\section{PERIOD DETERMINATION AND CLASSIFICATION}

The MACHO observations were carried out between 1992 and 2000 with the $1.27 \mathrm{~m}$ Great Melbourne telescope at Mount Stromlo Observatory, Australia. The telescope was equipped with a specificly designed camera, which gave a 0.5 square degree field of view. The observations were obtained in two non-standard bandpasses simultaneously: a 440-590 nm MACHO "blue" filter and a 590-780 nm MACHO "red" filter (Cook 1995). The typical number of observational points obtained is about 1200. Some of the data are publicly available on the MACHO website ${ }^{2}$, where one can select samples based on an automatic variability type classification. Using the web-interface, we individually downloaded light curves of all stars classified as eclipsing binaries, 6833 in total.

\footnotetext{
2 The MACHO variable star catalog is available at http://wwwmacho.mcmaster.ca
} 
However, it became obvious very quickly that the automatic classification was not perfect, as a large fraction of 'eclipsing binaries' turned out to be Cepheids, RR Lyrae stars or long-period variables. We therefore needed to re-classify all stars. Furthermore, besides the problems with the classification, we also found that the MACHO periods were incorrect for significant number of stars. For this reason, we redetermined periods for all the 6833 objects and classified them based on the light curve shapes. Since the MACHO light curves contain more blue points than red ones, we used the former for determining periods.

For an initial period determination, we used the Phase Dispersion Minimization method (PDM, Stellingwerf 1978). The method is based on a technique to minimize the sample variance of the phase diagram. It is calculated in the following way. The phase diagram for a given trial period is divided into $m$ segments, each containing $n_{j}(j=1 \ldots m)$ points. The variance for each segment is defined as $s_{j}^{2}=\sum_{i=1}^{n_{j}}\left(m_{i}-\bar{m}_{j}\right)^{2} /\left(n_{j}-1\right)$, where $\mathrm{m}_{\mathrm{i}}$ is the observed magnitude, $\overline{\mathrm{m}}_{\mathrm{j}}$ is the mean magnitude: $\bar{m}_{j}=\sum_{i=1}^{n_{j}} m_{i} / n_{j}$. The sample variance $s^{2}=\sum_{j=1}^{m} s_{j}^{2}$ is minimized to get the best period. We calculated 600 000 trial phase diagrams for each star, covering a wide range of periods between 0.085 days and 1000 days (from 0.001 cycles/day to 12 cycles/day, with equidistant steps in frequency).

However, this was not enough for determining the true periods. As commonly happens in finding periods from light curves of eclipsing binaries, a significant fraction of the PDM results were harmonics or subharmonics of the true period $\left(\mathrm{P}_{\mathrm{PDM}} / \mathrm{P}_{\text {true }}\right.$ was a ratio of small integers). To correct for this, we proceeded as follows. A visual inspection of every phase diagram showed whether the actual period was an alias or just slightly inaccurate. In the case of an alias, we multiplied the PDM period by different constants (in most cases by 2) until the shape of the curve was consistent with that of an eclipsing binary.

We next used the String-Length method Lafler \& Kinman 1965; Clarke 2002) to improve period determination. With this method, one calculates the total length of the phase diagram for any given period as the following sum: $\mathrm{SL}^{2}=\sum_{\mathrm{i}=1}^{\mathrm{n}-1}\left(\left(\mathrm{~m}_{\mathrm{i}+1}-\mathrm{m}_{\mathrm{i}}\right)^{2}+\left(\varphi_{\mathrm{i}+1}-\varphi_{\mathrm{i}}\right)^{2}\right), \quad$ where $\left\{\varphi_{\mathrm{i}}, \mathrm{m}_{\mathrm{i}}\right\}_{\mathrm{i}=1}^{\mathrm{n}}$ is a folded dataset sorted in phase; $\varphi_{\mathrm{i}}$ and $m_{i}$ are the phase and the magnitude of the observations taken at time $t_{i}$ (so that $\varphi_{i}=\left[\left(t_{i}-t_{0}\right) / P\right]$, where $t_{0}$ was chosen as an epoch of the deeper minimum, $\mathrm{P}$ is the period, [ ] is the fractional part). The best period here was the one that minimized $\mathrm{SL}^{2}$. In our case, we applied the SL method for 1000 periods within $\pm 1 \%$ of the best PDM period. An example of the period improvement is shown in Fig. 1] The typical period improvements resulted in a change in the 5-6th decimal place.

During the visual inspection of the phase diagrams we also made a rough classification of all 6833 variables. Based on the light curve shape, phased with the finally adopted periods, we placed each star into one of the following categories: Algol-type, $\beta$ Lyrae-type, $W$ UMa-type eclipsing binary, pulsating star (including RR Lyraes, Cepheids, Miras, etc.), non-periodic or multiply periodic, and unidentifiable. In cases of a pure sine-wave,
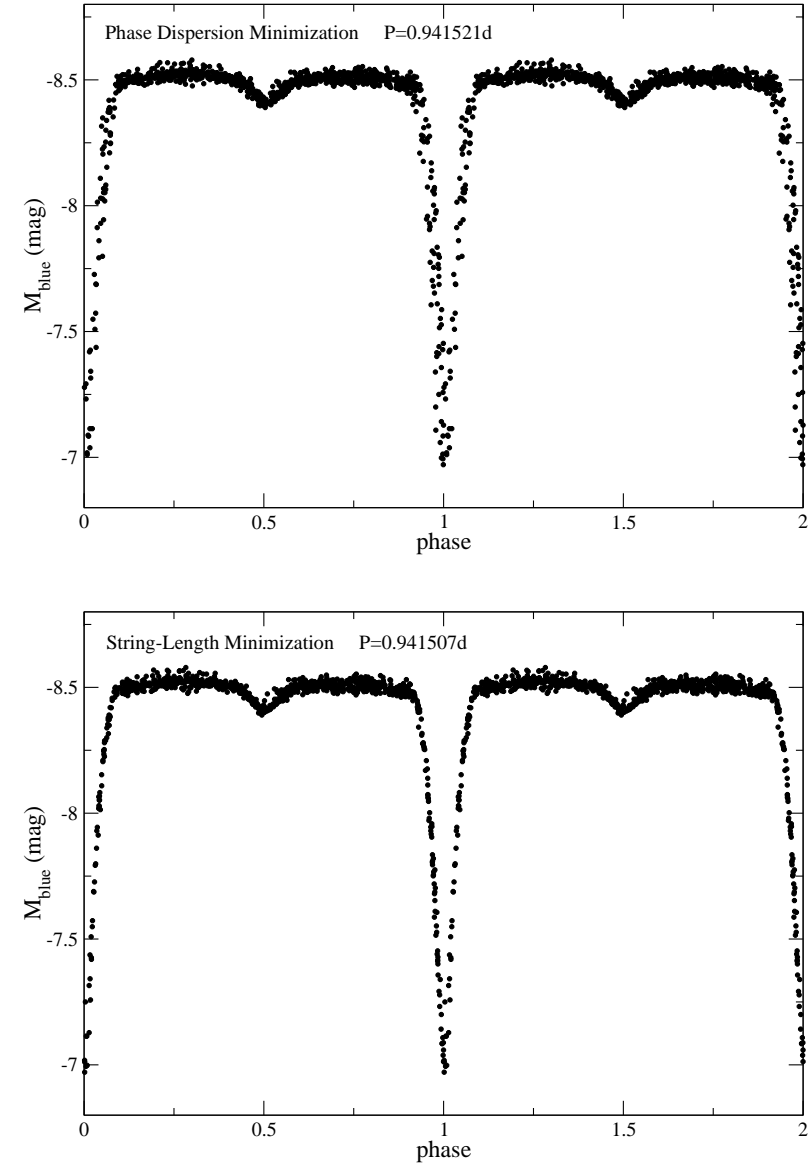

FIG. 1.- An example (81.9006.28) for improvement in period determination using PDM (top panel) and SL (bottom panel) methods.

which can be observed in a contact binary or a pulsating star, we compared the amplitudes of the MACHO blue and red phase diagrams. If there were noticeable color variations exceeding a few hundredth of a magnitude, we considered the star as pulsating variable.

After the whole procedure, we ended up with 3031 genuine eclipsing or ellipsoidal binaries, the rest belonging to other type of variables. The identification numbers, J2000 coordinates, calibrated colors and magnitudes, periods and epochs of minimum light for the eclipsing variables are listed in Table1 (available in full electronically). In the last column of the table, we note cases where a very faint secondary eclipse might possibly exist, which would result in a 1/2 ambiguity in the period. 12 of the 3031 systems have no periods because they showed signs of only one eclipse over the eight years of MACHO observations.

A comparison of our periods with those in the online MACHO catalog shows that there is a major disagreement. A plot of the ratios of the periods (Fig. 2) reveals an interesting structure. A minor fraction of the period ratios are scattered around 1, while the majority are dominated by the cases when the MACHO period is half of ours. Also, we identified a number of stars with period ratios at $1 / 3,1 / 4,1 / 5$, etc. The diagonal line in the lower part of the histogram corresponds to cases when the MACHO frequency $\left(1 / \mathrm{P}_{\mathrm{MACHO}}\right)$ is \pm 1 $\mathrm{c} / \mathrm{d}$ offset from the true frequency or one of its integer harmonics. 


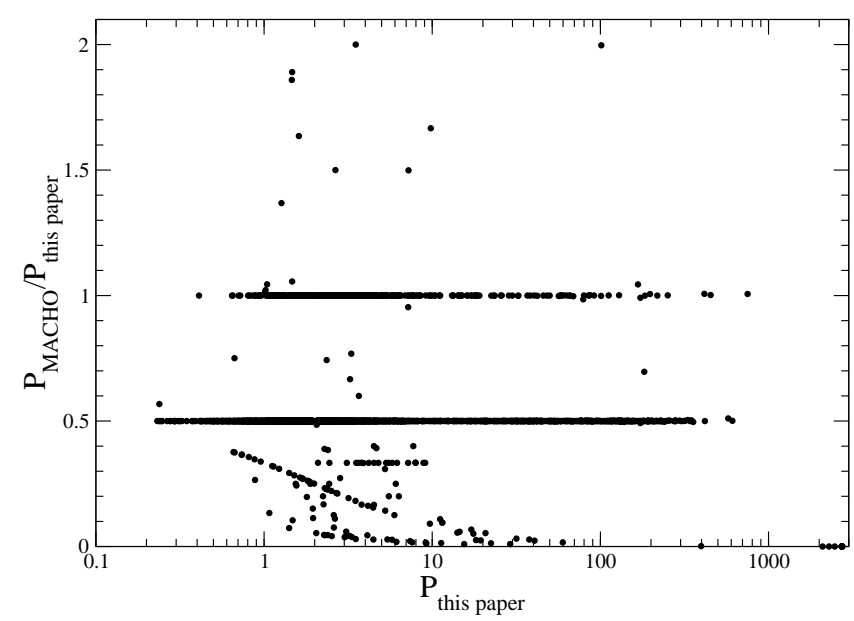

FIG. 2.- A comparison of periods presented in this study and those of the MACHO project.

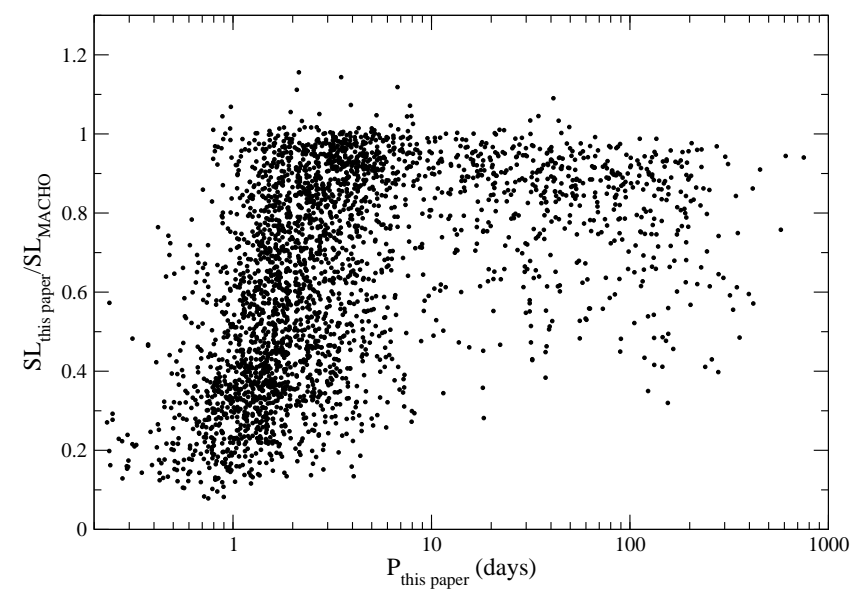

FIG. 3.- The ratio of the length of phase diagrams calculated with period in this paper to that using the MACHO period versus period of this paper.

To illustrate the improvement of periods in this paper compared to those in the online MACHO catalog, we performed the following statistical evaluation. For each star, we calculated the string-length using both our period and that of the $\mathrm{MACHO}$ online catalog. The ratio of the two lengths is plotted as a function of period in Fig. 3. We see a considerable improvement for all but a few stars; the points over 1.0 all refer to datasets with very few points, for which none of the statistics is well defined.

Ratios other than 0.5 occurred almost exclusively in stars with eccentric orbits, which causes a shift in the secondary minimum from 0.5 phase. These problems clearly illustrate that one has to be very careful when determining periods automatically for eclipsing binaries.

The histogram of the period ratios (Fig. 4) shows that roughly $16 \%$ of the periods agree. The true period turned out to be the double of the given MACHO period in about $78 \%$ of the binary sample, while the remaining $6 \%$ have other ratios. In Fig. [5] we show a few typical examples, plotting phase diagrams with the MACHO and our periods.

With the corrected phase diagrams, it became possible to re-classify the sample. As discussed above, pulsating and non-periodic variables were easily excluded,

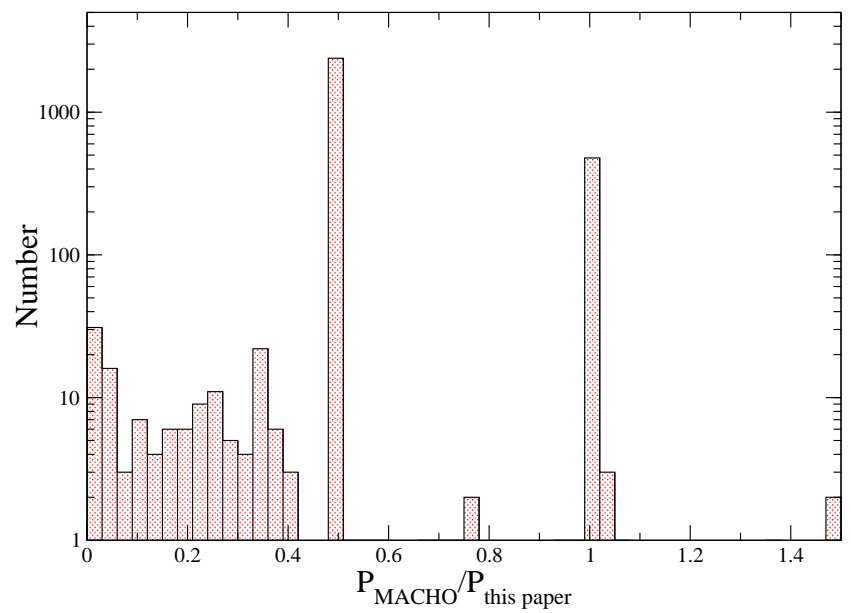

FIG. 4. - The histogram of period ratios presented in this study and by the MACHO project. Note the vertical axis is in logarithmic scale.

while eclipsing binaries were visually pre-classified as Algol (EA), $\beta$ Lyrae (EB) and W UMa (EW) type stars. However, this kind of classification contains some subjectivity, so we decided to use a more objective method for this purpose. Rucinski (1993) showed that light curves of W UMa systems can be quantitatively described using only two coefficients, $\mathrm{a}_{2}$ and $\mathrm{a}_{4}$, of the cosine decomposition $\sum \mathrm{a}_{\mathrm{i}} \cos (2 \pi \mathrm{i} \varphi)$. Pojmański (2002) tested the behavior of semidetached and detached configurations in the $\mathrm{a}_{2}-\mathrm{a}_{4}$ plane by decomposing theoretical light curves into Fourier coefficients. He found that in most cases contact, semi-detached and detached configurations can be distinguished quite accurately. We chose this method because it can be easily implemented for a large set of light curves.

Following the definitions by Pojmański (2002), we decomposed every light curve in the following form:

$$
m(\varphi)=m_{0}+\sum_{i=1}^{4}\left(a_{i} \cos (2 \pi i \varphi)+b_{i} \sin (2 \pi i \varphi)\right)
$$

where $m(\varphi)$ is the phased light curve, $m_{0}$ is the mean magnitude, while the zero point of the phase corresponds to the primary minimum. The resulting distribution in the $\mathrm{a}_{2}-\mathrm{a}_{4}$ plane, based on the MACHO blue data, is shown in Fig. 6. Using the boundary lines of Poimański (2002), we marked the contact (EC), semidetached (ESD) and detached (ED) configurations with different symbols.

The sample is dominated by bright main-sequence detached (1482 stars) and semidetached (937 stars) binaries, while a small fraction consist of contact systems (612 stars). Many of the latter are foreground Milky Way objects, as can be shown from their positions in the color-magnitude diagram (Section 3.2). We list the EC/ESD/ED classification for each star in Table 1.

\section{GENERAL PROPERTIES}

After the visual inspection, we identified 3031 stars as eclipsing binaries, which is about $44 \%$ of the downloaded sample. We emphasize that our sample probably does not contain all LMC eclipsing binaries observed by the MACHO project. We assume that there might be 
TABLE 1

Representative lines from the full Table 1 (available Electronically). The (*) Symbol in the Type Column Denotes FOREGROUND OBJECTS.

\begin{tabular}{|c|c|c|c|c|c|c|c|c|}
\hline ID number & $\begin{array}{c}\text { RA } \\
(\mathrm{J} 2000.0) \\
\end{array}$ & $\begin{array}{c}\text { Dec. } \\
(\mathrm{J} 2000.0) \\
\end{array}$ & $\begin{array}{c}\mathrm{V} \\
(\mathrm{mag}) \\
\end{array}$ & $\begin{array}{r}\mathrm{V}-\mathrm{R} \\
(\mathrm{mag}) \\
\end{array}$ & $\begin{array}{r}\text { Period } \\
(\text { days })\end{array}$ & $\begin{array}{c}\text { Epoch } \\
(2400000+) \\
\end{array}$ & Type & Note \\
\hline 47.1402 .116 & $04: 49: 21.544$ & $-68: 12: 31.70$ & 18.644 & -0.034 & 1.761188 & 50249.7483 & ESD & \\
\hline 47.1527 .178 & 04:49:28.952 & $-67: 55: 24.15$ & 19.223 & 0.725 & 12.556414 & 50244.6082 & $\mathrm{EC}$ & \\
\hline 47.1528 .41 & $04: 49: 34.577$ & $-67: 49: 48.88$ & 17.209 & -0.179 & 5.024733 & 50249.8034 & $\mathrm{ED}$ & \\
\hline 47.1529 .60 & $04: 49: 44.988$ & $-67: 48: 57.28$ & 18.005 & 0.574 & 112.073172 & 50252.7272 & ED & \\
\hline 47.1531 .32 & 04:49:51.948 & $-67: 41: 38.13$ & 16.842 & -0.159 & 0.459804 & 50249.9699 & $\mathrm{EC}$ & \\
\hline 47.1530 .30 & $04: 49: 52.060$ & $-67: 42: 09.84$ & 15.519 & -0.249 & 1.633056 & 50249.3579 & ED & \\
\hline 47.1649 .69 & $04: 50: 16.357$ & $-67: 53: 06.87$ & 17.396 & -0.153 & 1.453116 & 50250.0195 & ESD & \\
\hline 47.1645 .115 & $04: 50: 21.514$ & $-68: 07: 37.01$ & 18.048 & -0.163 & 1.711124 & 50249.3182 & ED & \\
\hline 47.1647 .143 & 04:50:33.106 & $-67: 58: 44.94$ & 18.440 & -0.186 & 1.620856 & 50250.1698 & ED & \\
\hline 47.1647 .285 & $04: 50: 34.347$ & $-67: 59: 24.49$ & 19.653 & 0.082 & 1.387449 & 50249.4512 & ESD & \\
\hline 11.9720 .84 & 05:40:08.972 & $-70: 15: 03.29$ & 17.506 & 0.953 & 4.607095 & 50248.2811 & $\mathrm{EC}(*)$ & \\
\hline
\end{tabular}

eclipsing binaries classified as other variable types (such as RR Lyraes, Cepheids, Semiregulars, etc.). For example, Faccioli et al. (2005) studied orbital circularization of LMC eclipsing binaries presenting a new sample of 4576 stars; however, that sample is not available to us. With this caveat, we discuss the main properties of the sample.

\subsection{Period distribution}

The most fundamental parameter of binary stars is the orbital period, whose distribution can be of great aid in understanding formation and evolution of close binaries. Eclipse detection is highly influenced by the orbital period, because the wider the separation of components, the longer the orbital period gets, implying that eclipses will be seen in narrower range of the inclination angle. Hence the chances to detect eclipses are much higher for short orbital periods. Well before the microlensing projects, Farinella \& Paolicchi (1978) and Giuricin et al. (1983) found a multi-modal period distribution in the Milky Way. Recent studies of eclipsing binaries in the Small and the Large Magellanic Clouds revealed an overall similarity in the period distributions, peaking between 1 and 2 days (Alcock et al. 1997; Wyrzykowski et al. 2003, 2004; Devor 2005).

For a direct comparison with the OGLE sample for the LMC and the SMC, we downloaded periods of eclipsing binaries from the OGLE internet archive ${ }^{3}$. Our period distribution (Fig. 7) is in a good agreement with that of the OGLE sample of LMC and SMC stars (Wyrzykowski et al. 2003, 2004): the majority of systems have short periods, peaking between 1 and 2 days, and roughly $20 \%$ of stars have periods longer than 10 days, which is consistent for both the SMC and the LMC.

The distribution shown in the upper panel of Fig. 7 is also similar to that of Devor (2005), which was based on the OGLE observations of the Galactic Bulge. However, we do not confirm his conclusion that the peak at $\sim 100$ days is due to pulsating red giant stars. While Devor (2005) used single filtered I-band data of the OGLE project, thus having no information on the color variations, the two-color MACHO observations clearly showed

\footnotetext{
${ }^{3}$ http://bulge.astro.princeton.edu/ ogle
}

that those long-period variables have only slight color changes, which can be explained by the non-uniform temperature distribution on the surface of the non-spherical components.

The lower panel of Fig. [7 shows the histogram of all eclipsing binaries in the General Catalog of Variable Stars (Kholopov et al. (1985-1988) with all the recent updates available for download from the GCVS website ${ }^{4}$ ). Compared to the upper panel of Fig. 7, the distributions are different in two period ranges. For shorter periods, there is an excess of stars in the GCVS, which are all short-period main-sequence binaries that have fainter absolute magnitudes than the MACHO limit in the LMC. On the other hand, for periods longer than 40 days, there is a lack of stars in the GCVS, which we interpret as caused by a selection effect: these systems need years of observations before classification and period determination, which was hardly possible before the development of automatic all-sky survey projects like the All-Sky Automated Survey (ASAS, Poimański 2002). It also implies that there are considerable number of bright long-period eclipsing binaries waiting for discovery.

To make further comparison, we took detached and semi-detached binaries in the Galaxy observed by the ASAS project and compared their period distribution with detached and semi-detached binaries in the SMC and LMC. In the upper panel of Fig. 8 we plotted the period distribution of orbital periods shorter than 10 days, which shows an overall similarity for the three galaxies. This diagram also shows the presence of a selection effect in the data: there is a noticeable dip at $\mathrm{P}=2 \mathrm{~d}$ in the histograms of the MACHO and the ASAS data, which reveals that stars with exactly 1 -d periods (i.e. the half of the true ones) were discarded during the initial analyses. For example, based on the shape of the histogram, about 50 MACHO eclipsing binaries with $\mathrm{P} \sim 2 \mathrm{~d}$ are missing from the sample, presumably due to deliberate exclusion from the data.

In the lower panel of Fig. 8 we plot the cumulative period distribution for the three samples. While the LMC and SMC samples have virtually indistinguishable distributions, the Milky Way shows an excess of stars around

\footnotetext{
${ }^{4}$ http://www.sai.msu.su/groups/cluster/gcvs/gcvs/
} 

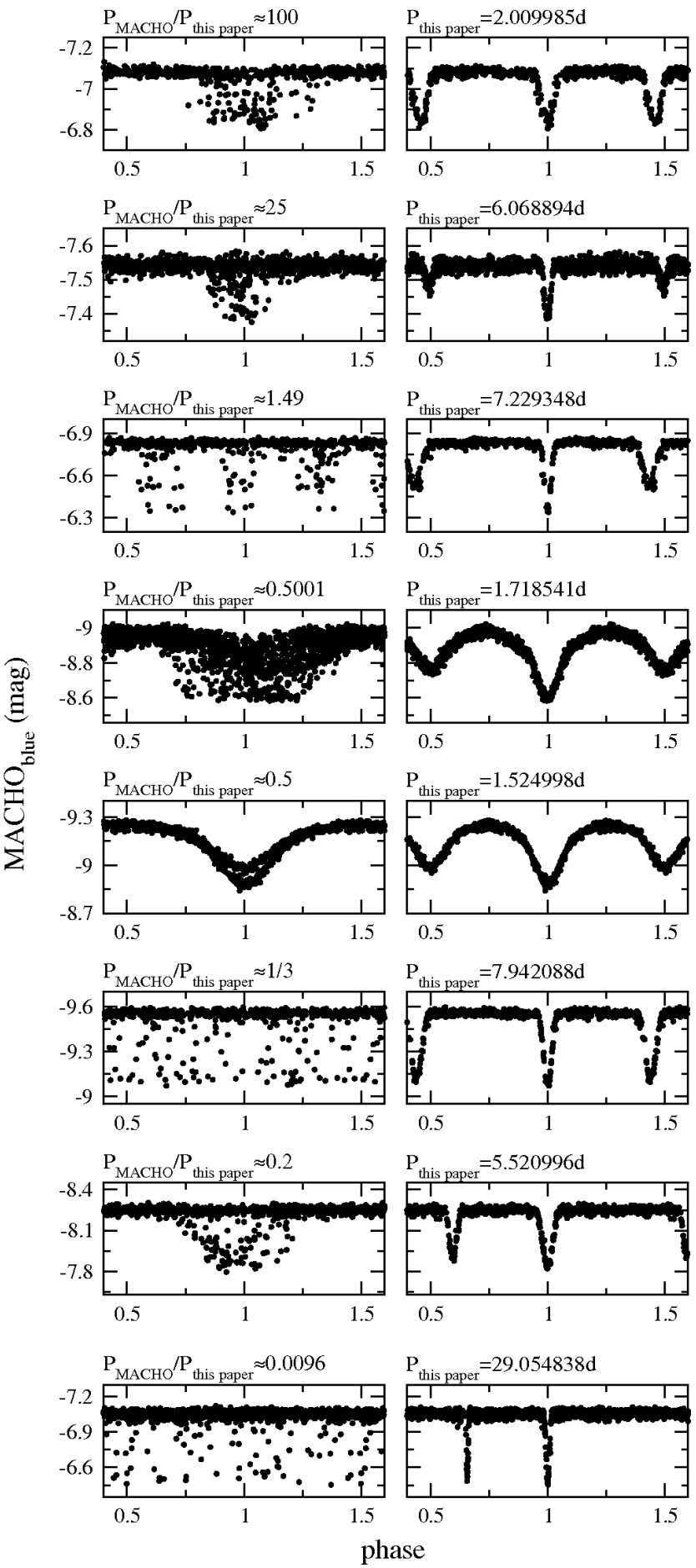

FIG. 5.- Examples of typical period ratios. In each case, the left panel shows the data phased with the MACHO period, while the right panel shows the final phase diagram with our period.

10 days. A two-sample Kolmogorov-Smirnov test showed that the difference between the LMC and the SMC samples is insignificant, which confirms that the MACHO and OGLE selections of detached and semi-detached binaries were similarly complete. On the other hand the probability that the ASAS stars have the same distribution is only $10^{-11} \%$. This is a simple consequence of the different sampling: while MACHO and OGLE data represent magnitude-limited samples in absolute magni-

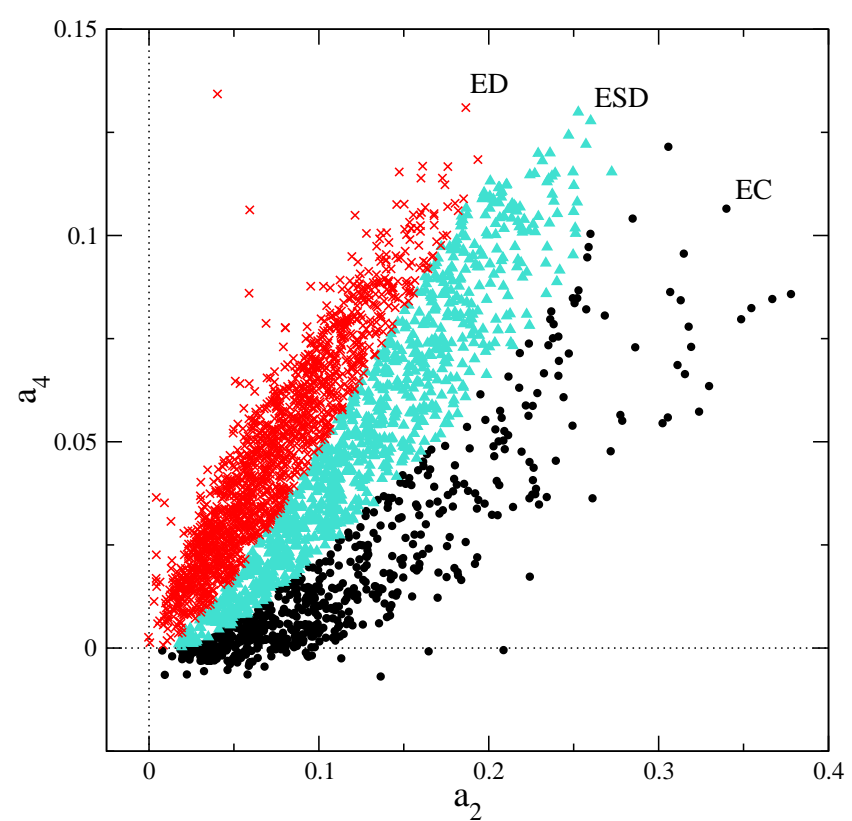

Fig. 6.- Classification of LMC eclipsing binaries in the Fourier coefficients plane $\mathrm{a}_{2}-\mathrm{a}_{4}$.
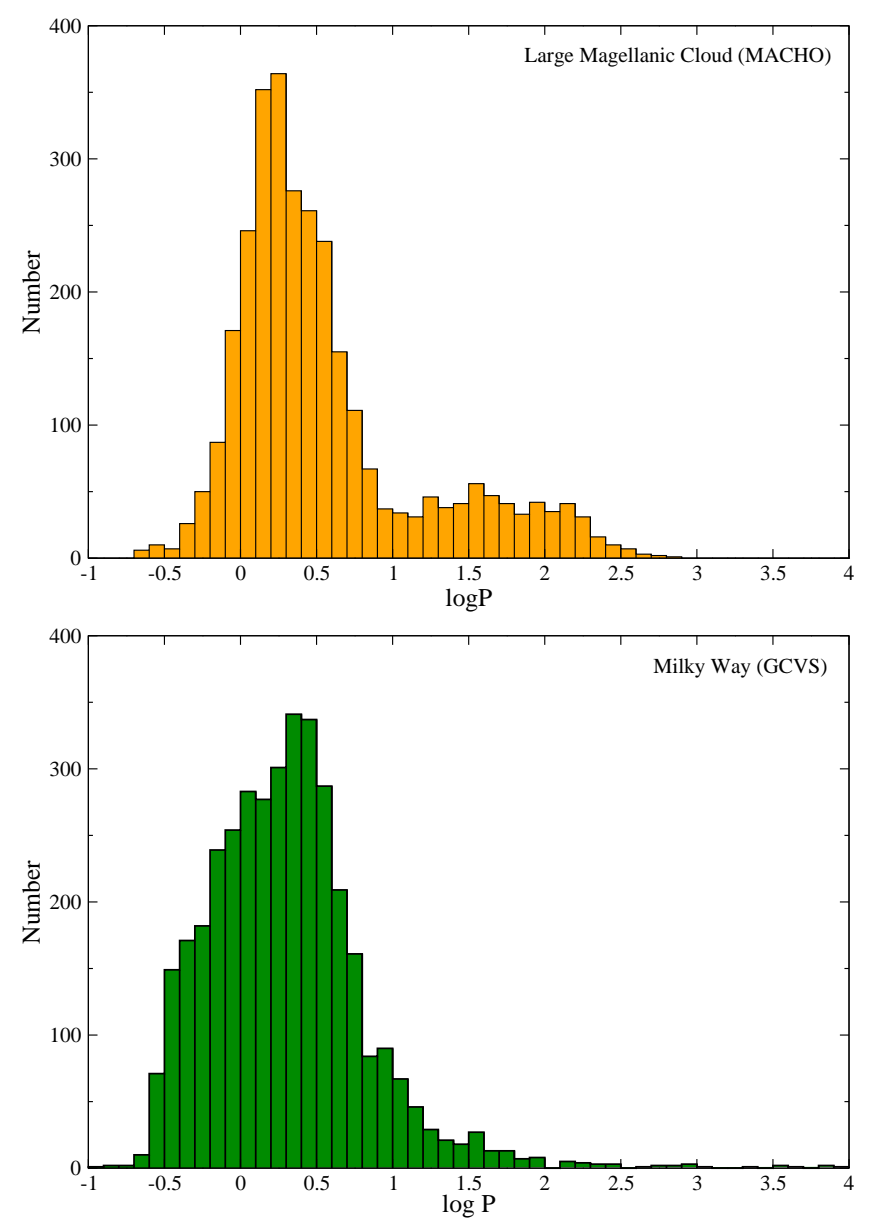

FIG. 7.- Upper panel: period distribution of eclipsing binaries in the MACHO database. Lower panel: histogram of orbital periods of eclipsing binaries in the General Catalog of Variable Stars. 

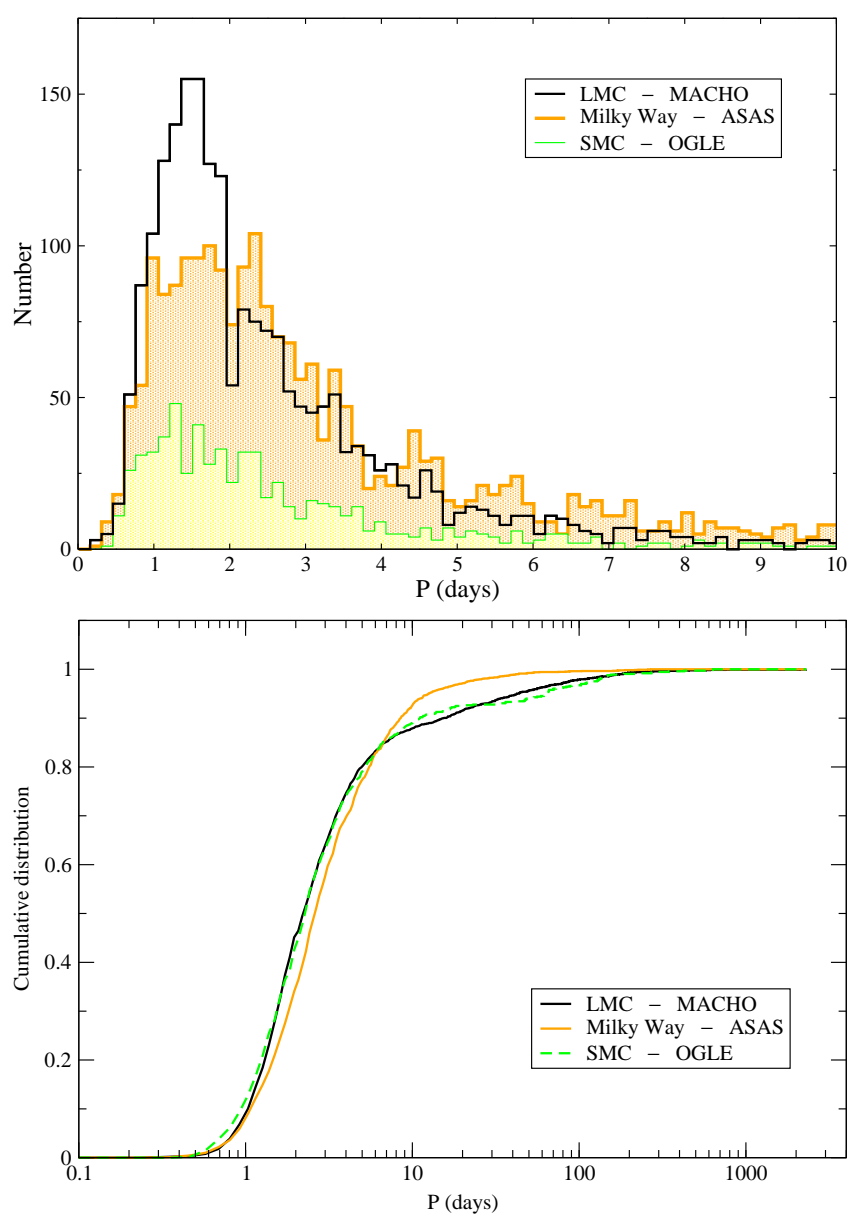

FIG. 8.- Upper panel: Orbital period distributions of detached and semi-detached eclipsing binaries in the Milky Way and the Magellanic Clouds. Lower panel: The normalized cummulative distributions.

tudes, covering the brightest $\sim 6$ mag of the CMD, the ASAS database contains a broad mixture of stars in the Milky Way with a much wider range in absolute magnitude.

It is also apparent in Fig. 8 that, although the longperiod tails of the ASAS and MACHO histograms overlap very well, the short-period peak in the LMC data is higher because of the different distributions of stars in the samples. Whereas the MACHO data cover the upper part of the Hertzsprung-Russell diagram with a relatively bright limit in absolute magnitude, the ASAS sample contains a broader mixture of stars in the galactic neighborhood, so that short-period B-type systems have a smaller relative contribution.

\subsection{The Color-Magnitude Diagram and Period-Luminosity Relations}

In order to construct the Color-Magnitude Diagram (CMD), we converted the observed MACHO blue and red magnitudes into Kron-Cousins $\mathrm{V}$ and $\mathrm{R}$ using the equations derived by Alcock et al. (1999). The resulting diagram, after translating apparent magnitudes outside the eclipses to absolute magnitudes using $\mu(\mathrm{LMC})=18.50$, is shown in Fig. 9. We also show stellar evolutionary tracks by Castellani et al. (2003). Most of the stars are moderately massive near-main sequence stars, while the red giant branch of evolved stars is also clearly recognis-

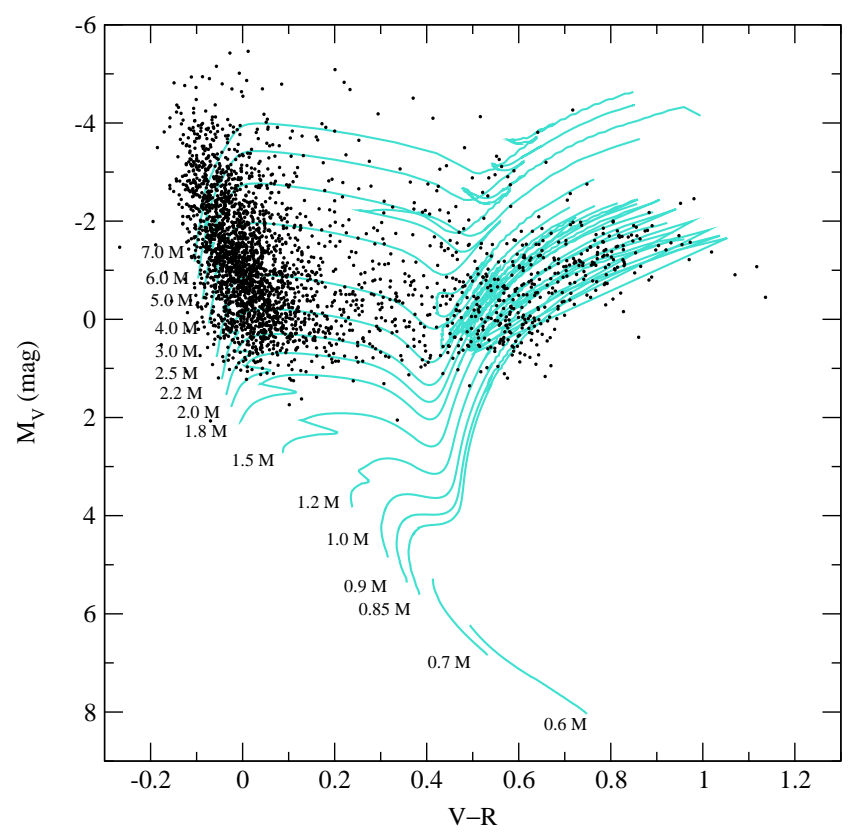

FIG. 9.- The color-magnitude diagram of LMC eclipsing binaries with metal-poor evolutionary tracks of single stars overlaid (models taken from Castellani et al. (2003)).

able. The latter is dominated by first-ascent red giants and a few Asymptotic Giant Branch stars (Alcock et al. 2000).

In Fig. 10 we plot the CMD for four orbital period ranges. These plots were used for cleaning the sample of the foreground objects, adopting the following simple considerations. First, the spread of distances of eclipsing binaries in the LMC is negligible compared to the distance to the LMC. This means that we can determine the absolute magnitude for any given object within \pm 0.1 mag (e.g. Nikolaev et al. 2004; Lah et al. 2005). Second, we assumed that the mean position of a binary star in the CMD can be approximated by the location of the brighter component. In the case of two identical components, this assumption means a 0.75 mag fainter absolute magnitude, however, as we proceeded, this was still a useful simplification in estimating foreground contamination.

Theoretical evolutionary tracks give the basic physical parameters of a single star, as well as color and magnitude information. From the physical parameters we can calculate the minimum orbital period for any given mass, temperature and luminosity, applying simple relations. Combining the Stefan-Boltzmann law: $L / L_{\odot}=\left(R / R_{\odot}\right)^{2}\left(T / T_{\odot}\right)^{4}$ and Kepler's third law: $a^{3} / P^{2}=G\left(M_{1}+M_{2}\right) / 4 \cdot \pi^{2}$, we can calculate the minimum orbital period of a system for two extreme cases:

Case 1.: two identical components, for which the minimum orbital period occurs when $a=2 R$ and $M_{1}+M_{2}=$ $2 M$; the minimal orbital period is

$$
P_{\min }(1)=4 \pi \sqrt{\frac{R^{3}}{G M}}
$$

Case 2.: a negligible secondary component, for which the minimum orbital period occurs when $a=R$ and $M_{1}+M_{2}=M$; the minimum orbital period is 

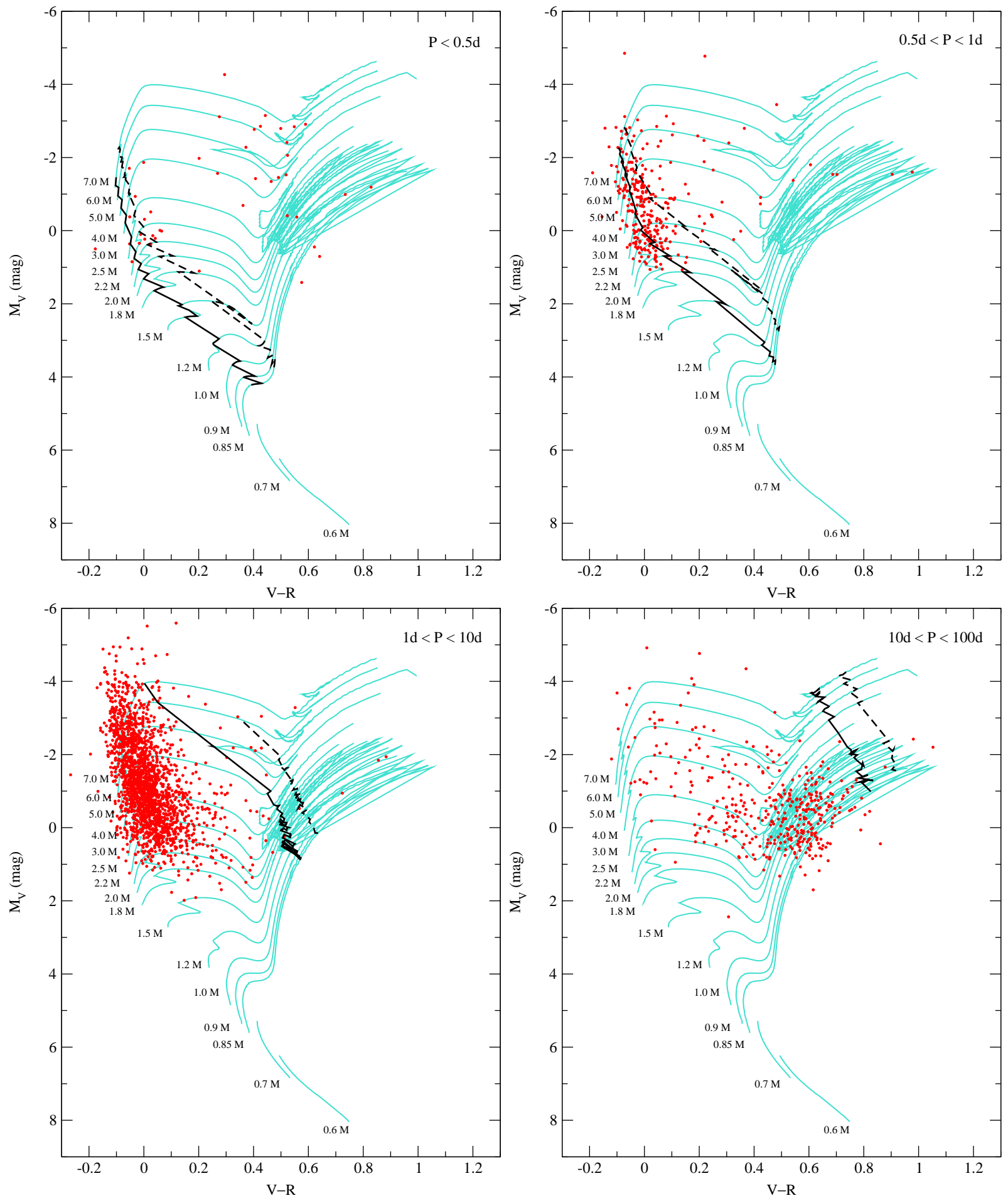

FIG. 10. - Eclipsing binaries in four period ranges and estimated locations of the minimum orbital periods. Stars upper and right of the dashed lines have positions that are not compatible with the LMC membership. 


$$
P_{\min }(1)=2 \pi \sqrt{\frac{R^{3}}{G M}}
$$

( $M$ is the mass of a model point of the isochrone, while $R$ is calculated from $L$ and $T$ ).

Using Eq. (2) and Eq. (3) and theoretical stellar evolutionary tracks, we can determine contours in the CMD, on which the minimum orbital period has a given value. This is shown in the four panels of Fig. 10 by the thick solid lines (Case 1) and dashed lines (Case 2). As representative limits, we determined the locations where the minimum orbital period is $0.5,1,10$ and 100 days.

We have selected the foreground objects as follows. As Fig. 10 shows, moving up and right in the CMD, the minimum orbital period gets longer. Therefore, if a star with $P_{\text {orb }} \leq 0.5 \mathrm{~d}$ is located above the limiting line of $P_{\min }=0.5 \bar{d}$, it must be in the foreground of the LMC. This is shown in the upper left panel of Fig. 10. As expected, most of these short-period reddish eclipsing binaries are Galactic W UMa-type stars, many magnitudes above the calculated period lines (which means the adopted simplifications do not affect the conclusions).

For longer periods, only a few stars are clearly foreground objects (most notably in the upper right panel of Fig. 10), while for the majority, positions in the CMD are compatible with the LMC membership. We flagged all obvious foreground stars, being those located at least $1 \mathrm{mag}$ above the dashed lines, in the last column of Table 1 (54 stars in total).

Finally, we briefly examine the period-luminosity $(\mathrm{PL})$ relation of stars in the sample, for which we found nearinfrared K-magnitudes in the 2MASS Point-Source Catalog. In Derekas et al. (2006) we already discussed how the red giant eclipsing binaries/ellipsoidal variables can be fitted with a simple model using Roche-lobe geometry. Here we examine the correlation between the period and the K-magnitude for the detached and semi-detached binaries. This is shown in Fig. 11, where the ED/ESD/EC classes are plotted with different symbols. As expected, detached binaries are spreaded uniformly, while longer period semi-detached systems may follow a loose correlation (the correlation coefficient for $\mathrm{P}>10 \mathrm{~d}$ is $\sim 0.58$ ), but it is not as tight as for long-period contact binaries (whose correlation coefficient is $\sim 0.86$ ). Systems further away from the main correlation line might be foreground stars left unidentified as such in the CMD analysis.

\section{SUMMARY}

In this paper we present the analysis of online light curves of eclipsing binaries in the Large Magellanic Cloud monitored by the MACHO project. We downloaded the data of all 6833 stars classified as eclipsing binaries from the Variable Star Catalog Retrieval form of the project.

We re-determined the periods for every star and reclassified all stars based on their light curve shape. As a result, 3031 stars remained as eclipsing binary, while the rest of the sample were RR Lyrae stars, Cepheids or long-period pulsators. For the binary sample, we showed that roughly $16 \%$ of the periods agreed with those given in the catalog. For almost three-quarter of the sample, the catalog periods turned out to be the half of the real ones.

We calculated the period histogram, which shows bi-

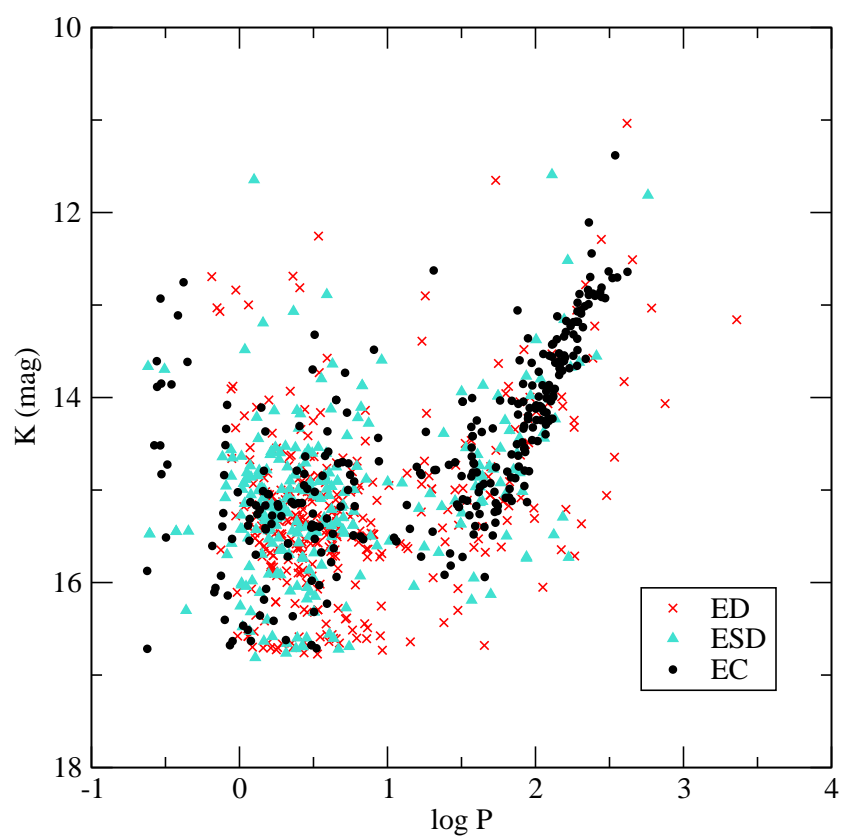

FIG. 11.- Period-K magnitude relation for eclipsing binaries in the LMC.

modal feature on logarithmic scale. The maximum of the distribution is at binaries with periods between 1 and 2 days, while roughly $20 \%$ of the sample (about 600 stars) have period between 10 and 200 days. We compared our period distribution for detached eclipsing binaries, with those of the SMC and the Milky Way and we found a good agreement in the most common periods in the studied galaxies.

For a more objective classification, we used cosine decomposition of the light curves into Fourier coefficients, where contact, semi-detached and detached configurations can be distinguished in $\mathrm{a}_{2}-\mathrm{a}_{4}$ plane. The sample is dominated by bright main-sequence detached and semi-detached systems, while a small fraction consists of contact binaries. However, many of the latter are foreground Milky Way objects, which can be shown from their position in the color-magnitude diagram. Most of the stars with periods shorter than 0.5 days are galactic W UMa systems. For longer period systems, we found a few obviously foreground objects and they are flagged in the last column of Table 1 .

The presented database opens up a new avenue into using the MACHO database for studying secular variations over a time-scale of a decade. This includes a search for systems with apsidal motion (recognizable through phase shifts of the primary and secondary minima in the opposite direction), tertiary components (indicated by cyclic phase shifts of the primary and secondary minima in the same direction) and eclipsing binaries with pulsating components (revealed by periodic secondary brightness fluctuations). The latter would be particularly interesting if evidence for tidally induced oscillations could be found in a larger sample of stars. With the OGLE-III project still taking data, after identifying these theoretically important test objects, it would become possible to extend the full time-coverage of observations up to 14-15 years. In a companion paper we will discuss the measured period changes for the whole sample of 3031 
eclipsing binaries. To help the interested researchers, we have collected the original MACHO blue and red light curve data in a single compressed data file that can be accessed as an electronic appendix to this paper.

We are greatful to the anonymous referee for his/her comments that improved the paper.

AD is supported by an Australian Postgraduate Award of the Australian Department of Education, Science and Training. LLK is supported by a University of Sydney Postdoctoral Research Fellowship. This work has been supported by the Australian Research Council.
The NASA ADS Abstract Service was used to access data and references.

This paper utilizes public domain data obtained by the MACHO Project, jointly funded by the US Department of Energy through the University of California, Lawrence Livermore National Laboratory under contract No. W-7405-Eng-48, by the National Science Foundation through the Center for Particle Astrophysics of the University of California under cooperative agreement AST8809616, and by the Mount Stromlo and Siding Spring Observatory, part of the Australian National University.
Alcock, C., et al., 1993, ASPC, 43, 291

Alcock, C., et al., 1997, AJ, 114, 326

Alcock, C., et al., 1999, PASP, 111, 1539

Alcock, C., et al., 2000, AJ, 119, 2194

Aubourg, E., et al., 1995, A\&A, 301, 1

Castellani, V., Degl'Innocenti, S., Marconi, M., Prada Moroni, P. G., \& Sestito, P., 2003, A\&A, 404, 645

Cook, K. H., Alcock, C., Allsman, H. A., et al. 1995, in

Astrophysical Applications of Stellar Pulsation, ed. R. S.

Stobie, \& P. A. Whitelock, ASP Conf. Ser., 83, 221

Clarke, D., 2002, A\&A, 386, 763

Derekas, A., Kiss, L. L., Bedding, T. R., Kjeldsen, H., Lah, P., \& Szabó, Gy. M., 2006, ApJ, 650, L55

Devor, J., 2005, ApJ, 628, 411

Faccioli, L., Alcock, C., Cook, K., Prochter, G., \& Syphers, D., 2005 , in Tidal evolution and oscillations in binary stars, ASP Ser., ed. A. Claret, A. Gimnez, \& J.-P. Zahn, 333, 75

Farinella, P., \& Paolicchi, P., 1978, Ap\&SS, 54, 389

Fitzpatrick, E. L., Ribas, I., Guinan, E. F., DeWarf, L. E., Maloney, F. P., \& Massa, D., 2002, ApJ, 564, 260

Giuricin, G., Mardirossian, F., \& Mezzetti, M., 1983, A\&A, 119, 218

Guinan, E. F., et al., 1998, ApJ, 509, 21

Johnson, A., Whelan, D., Edinger, B., Bailey, B., Smith, K., Malmrose, M., \& Palen, S. E., 2004, BAAS, 36, 740

Kholopov et al. 1985-1988, General Catalogue of Variable Stars, Vol. III, Nauka, Moscow

Lah, P., Kiss, L. L., \& Bedding, T. R., 2005, MNRAS, 359, 42L

\section{REFERENCES}

Lafler, J., \& Kinman, T. D., 1965, ApJS, 11, 216

Maceroni, C., \& Rucinksi, S. M., 1999, AJ, 118, 1819

Mazeh, T., Tamuz, O., North, P., 2006, MNRAS, 367, 1531

Michalska, G., \& Pigulski, A., 2005, A\&A, 434, 89

Nikolaev, S., Drake, A. J., Keller, S. C., Cook, K. H., Dalal, N. Griest, K., Welch, D. L., \& Kanbur, S. M., 2004, ApJ, 601, 260

Paczyński, B., Szczygiel, D., Pilecki, B., \& Pojmański, G., 2006, MNRAS, 368, 1311

Palen, S., \& Armstrong, J. C., 2003, BAAS, 35, 1222

Pojmański, G., 2002, Acta Astron., 52, 397

Ribas, I., et al., 2000, ApJ, 528, 692

Ribas, I., Fitzpatrick, E. L., Maloney, F. P., Guinan, E. F., Udalski, A., 2002, ApJ, 574, 771

Rucinski, S. M., 1993, PASP, 105, 1433

Rucinski, S. M., 1997a, AJ, 113, 407

Rucinski, S. M., 1997b, AJ, 113, 1112

Rucinski, S. M., 1998, AJ, 115, 1135

Rucinski, S. M., \& Maceroni, C., 2001, AJ, 121, 254

Salaris, M., \& Groenewegen, M. A. T., 2002, A\&A, 381, 440

Stellingwerf, R. F., 1978, ApJ, 224, 953

Tamuz, O., Mazeh, T., North, P., 2006, MNRAS, 367, 1521

Udalski, A., Kubiak, M., \& Szymański, M., 1997, Acta Astron., 47,319

Wyithe, J. S. B., \& Wilson, R. E., 2001, ApJ, 559, 260

Wyrzykowski, L., et al., 2003, Acta Astron., 53, 1

Wyrzykowski, L., et al., 2004, Acta Astron., 54, 1 